\title{
Desmitificado el voto de los mexicanos en el exterior: retos, falta de voluntad y otras realidades
}

\author{
Manlio César Correa Alcántar* y David Rocha Romero**
}

\begin{abstract}
Resumen
En 2006, el Pew Hispanic Center encontró que sólo un tercio de los inmigrantes mexicanos tenía credencial de elector. Seis años después, una encuesta a sesenta y ocho migrantes en el sur de California arrojó resultados parecidos. Desde que se comenzó a diseñar el voto extraterritorial hace 14 años, las estrategias basadas en cálculos y estimaciones que dieron como resultado el voto postal no han sido del todo certeras, no obstante el escenario favorable para incrementar la participación electoral de los inmigrantes. El presente trabajo sugiere vías para que este escenario sea aprovechado.
\end{abstract}

\begin{abstract}
In 2006, the Pew Hispanic Center found that only a third of Mexican immigrants had voting credentials. Six years later, a survey to sixty eight Mexican migrants in Southern California found similar results. In fourteen years since the conception of the expatriate vote strategies based on calculations and estimates that resulted in postal vote have not been entirely accurate, despite the existence of a propitious scenario. This article points fare to several ways in which this scenario can be taken advantage of.
\end{abstract}

Palabras clave: voto transnacional, migración, democracia, Estados Unidos, México. Keywords: transnational vote, migration, democracy, United States, Mexico.

* Maestro en Estudios Latinoamericanos por la Universidad Estatal de San Diego, California, Estados Unidos. Es fundador y director del Instituto Binacional de las Fronteras (IBF), San Diego, California, Estados Unidos.

* Doctor en Ciencias Políticas y Sociales con orientación en Relaciones Internacionales por la Universidad Nacional Autónoma de México y profesor titular B de la Facultad de Economía y Relaciones Internacionales de la Universidad Autónoma de Baja California, Tijuana, México. Es miembro del Sistema Nacional de Investigadores.

La aparición de los autores corresponde estrictamente a un orden alfabético. Los autores expresan su reconocimiento a los revisores anónimos de este trabajo por sus certeras observaciones y sugerencias, que contribuyeron a mejorar los planteamientos contenidos en el texto. 
E electoral de los inmigrantes mexicanos en Estados Unidos: la transición a la democracia mexicana, el interés de clubs de oriundos por asuntos públicos en sus lugares de origen y el interés del gobierno federal y gobiernos estatales por los inmigrantes. Sin embargo, dicho escenario no se está aprovechando, pues la falta de credenciales de elector limita de manera importante esta participación.

Las fuerzas políticas encargadas de diseñar el sistema electoral (Colomer, 2004: 31), no han cambiado sustantivamente su forma de pensar el voto extraterritorial para presidente de México desde la elección presidencial del 2006. En 1998, una comisión de expertos del Instituto Federal Electoral $(\mathrm{IFE})^{1}$ estimaba que 1.5 millones de mexicanos cumplían los requerimientos mínimos para votar desde Estados Unidos. Algunos de sus funcionarios declaraban que no existían inconvenientes técnicos para la credencialización en el extranjero. Se podría credencializar a los mexicanos que vivían en el país vecino como se había hecho con millones de mexicanos en el territorio nacional. ${ }^{2}$ Sin embargo, parecería que para entonces, el IFE sólo contemplaba a los mexicanos documentados que emigraron con credencial de elector, olvidando a millones de indocumentados, aquellos que se fueron antes de los 18 años y no han regresado a tramitar su credencial, otros que la dejaron en México o los que simplemente la perdieron. Se pensó que la mezcla de lealtad, nacionalismo y añoranza ayudaría a resolver los problemas legales, técnicos, financieros y de traslado que implica tramitar la credencial en México.

En la elección de 2012, al igual que en la de 2006, hubo una escasa participación electoral de los migrantes. Para algunos, esta situación representa un dispendio de recursos, pues consideran como innecesaria la extensión más allá de las fronteras del sistema electoral, lo que podría llevar a la desaparición del voto de los mexicanos en el extranjero. Sin embargo, vale la pena replantear mecanismos para incrementar tal participación, sobre todo si ya se dio el primer paso para escuchar a los que se fueron pero que siguen importando y aportando a México.

1 Organismo público autónomo encargado de organizar las elecciones federales desde 1990.

2 http://www.huellasmexicanas.org/alejandra/voto/InformeVoto-Mexicanos-Extranjero.pdf. Revisado, 10 de febrero de 2012. 
El presente trabajo tiene como objetivo mostrar que la escasa votación se debió a que sólo un tercio de los migrantes tenía credenciales de elector, -esto a pesar de que existe un mayor interés en votar- aunado a complicaciones de logística, tiempos y formas para inscripciones en las votaciones, así como al poco aprovechamiento de un escenario propicio para fomentar la participación electoral. Para poder promover un debate fructífero para los siguientes años, se deben repensar nuevas leyes, modalidades y estrategias que permitan expandir el sufragio e incrementar la igualdad que pretende la joven democracia mexicana. Por otro lado, se aborda la limitada representación política que tiene el Estado mexicano entre la comunidad migrante, a través del porcentaje de Matrículas Consulares de Alta Seguridad (MCAS) ${ }^{3}$ existentes en la comunidad. El 54 por ciento no cuenta con este documento, que se obtiene por medio de los consulados para identificarse y hacer uso de algunos servicios, como el bancario. Esto se menciona para reflexionar sobre la posibilidad de que este documento sustituya a la credencial de elector.

El artículo analiza los porcentajes de credenciales de elector y de MCAS en la Encuesta de Mexicanos Residentes en Estados Unidos en el Voto Ausente en Elecciones Mexicanas, Survey of Mexicans Living in the U.S. on Absentee Voting in Mexican Elections, realizada en 2006 por el Pew Hispanic Center, y se le compara con la Encuesta sobre Credenciales de Elector y Matrículas Consulares (MCAS) en el Sur de California realizada a sesenta y ocho mexicanos residentes en el sur de California, que se levantó durante octubre de 2011 y febrero de 2012. En ésta, resultaron los mismos porcentajes de tenencia de credenciales de elector y MCAS que en 2006.

Han sido pocos y con limitaciones estadísticas, los instrumentos utilizados por las autoridades electorales para saber cuántas credenciales de elector hay entre la comunidad migrante mexicana. Es una tarea complicada desarrollar el instrumento de medición estadísticamente representativo y aplicarlo, no sólo por los costos, sino por las dificultades intrínsecas a aplicar cuestionarios a indocumentados, toda vez que los sentimientos antiinmigrantes y el incremento de las deportaciones inhiben la exposición pública. El miedo es latente y la información se restringe. En Estados Unidos se considera que los cambios sociales y tecnológicos han incrementado las dificultades para contactar potenciales participantes de las encuestas (The Pew Research Center for the People and the Press, 2012). En la encuesta

3 Tarjeta otorgada por los consulados mexicanos que algunas oficinas de gobiernos locales estadounidenses e instituciones bancarias, entre otras, aceptan como instrumento de identificación. 
levantada por el Pew Hispanic Center antes referida, 987 personas aceptaron contestarla y 1017 la rechazaron, es decir, el 50.74 por ciento. Este es un porcentaje elevado si lo comparamos con porcentajes de rechazo a encuestas electorales en México (Grupo Reforma 2012). ${ }^{4}$ Por ello, conscientes de estas limitaciones, proponemos el porcentaje de credenciales de elector como limitante de la participación electoral en nuestra investigación para colocarlo en el debate académico y político. Un tercio de mexicanos con credenciales del IFE y más de la mitad sin matrículas consulares frente al amplio interés en votar, pueden ser situaciones que se estén reproduciendo a lo largo de todo el territorio estadounidense.

Generalmente se considera que la participación en el voto extranjero es limitada en los países que lo permiten, esto se debe en gran medida a factores políticos, administrativos, institucionales y financieros (Navarro, Morales y Gratschew, 2007: 32). Si comparamos con otros contingentes de migrantes, la participación electoral de los mexicanos en Estados Unidos no es atípica. Por ejemplo, hay 50 mil argentinos inscritos en el Registro de Electores Residentes en el Extranjero, una cifra baja si se compara con los 806 mil que se estima residen en el extranjero. En las elecciones de 2011 sólo 7649 acudieron a votar, el 15 por ciento (Emmerich y Moreira, 2013: 35). Si las autoridades electorales mexicanas apuestan a la media mundial de participación electoral desde el extranjero, no hay justificación para incrementar el voto. Sin embargo, existe un escenario político, que bajo los procedimientos adecuados, permitiría incrementarlo.

El trabajo está dividido en tres partes. La primera presenta la teoría de la asimilación fragmentada y transnacionalismo que sirven como premisas al argumento de que la comunidad inmigrante mexicana en Estados Unidos tiene importantes intereses políticos en sus comunidades de origen. Después, se presenta la metodología utilizada y los hallazgos de la encuesta levantada en el sur de California y su comparación con la del Pew Hispanic Center de 2006. En la segunda parte se discute la importancia del voto mexicano desde el extranjero y se analizan tres elementos que conforman lo que llamamos el escenario propicio para incentivar el aumento de la participación electoral. La tercera parte presenta las conclusiones a partir de la idea de que este escenario es desaprovechado.

4 En una encuesta sobre preferencias electorales en el Distrito Federal, levantada en junio de 2012 por el Grupo Reforma, un mes antes de las elecciones presidenciales, la tasa de rechazo fue de 38 por ciento. 
La asimilación de los inmigrantes mexicanos en Estados Unidos no ha sido voluntaria sino una obligación histórica para de tratar de sobrevivir, lo que genera una tensión permanente ya que la asimilación completa es impedida por la sociedad dominante a grupos racialmente diferentes (Johnson, 1997: 1278; Portes y Rumbaut, 2010: 30). Por esto, algunos han llamado a Estados Unidos una nación racista, que ha establecido distinciones históricamente acentuadas entre blancos, indios, negros, asiáticos y mexicanos (Huntington, 2004: 77). A pesar de esta contradicción, sectores antiinmigrantes culpan a los grupos minoritarios de rehusarse a asimilarse (Johnson, 1997: 1279; Huntington, 2004).

La asimilación entre la comunidad inmigrante se ha dado en diferentes grados dependiendo de diversas circunstancias. Empero, una característica de los migrantes mexicanos es que no están integrados a procesos políticos en la sociedad de destino (Johnson, 1997: 1285). Puede ser que esto se encuentre en proceso de cambio, sobre todo después del triunfo presidencial de Obama. No obstante, la estrechez de la integración política que se deriva del acceso a la ciudadanía, hace repensar el debate sobre la asimilación de procesos de incorporación a las sociedades de destino y a procesos de integración de las sociedades de origen y destino. La reflexión sobre integración ha dado como consecuencia la perspectiva del transnacionalismo, lo que ha provocado un análisis más allá de las fronteras de los Estados nacionales (Vertovec, 2003: 353). Alarcón, Escala y Odgers (2012: 43) han propuesto que los vínculos entre individuos que migran y sus lugares de origen no se volverán frágiles con el tiempo sino que pueden coexistir con una integración exitosa. Desde esta perspectiva, la migración ya no sería vista como un proceso de una sola vía (Levitt and Waters, 2002: 5), sino como un proceso transnacional. Para el caso de la migración mexicana, su creciente asentamiento facilita un patrón transnacional (Roberts, Reanne and Lozano-Ascencio, 2003: 45) que involucra a individuos junto con sus redes sociales, sus comunidades y estructuras institucionales más amplias como gobiernos locales y nacionales (Portes, Guarnizo y Landolt, 2003:19). La importancia de movimientos migratorios como éste ha llevado a extender el estudio del transnacionalismo (Emmerich y Moreira, 2013: 28).

$\mathrm{Al}$ sustentar el planteamiento transnacional, se encuentra que los migrantes de países democráticos asumen en mayor medida que tienen derechos políticos en el nuevo país, por ejemplo, al agilizar su naturalización. Por el contrario, quienes provienen de países no democráticos son más 
proclives a asumir que no tienen derechos en la nueva sociedad y muchos se mantienen al margen de la política. Sin embargo, éstos mantienen interés por la política de su país de origen, y si la fragilidad política como extranjeros los vuelve débiles y tímidos frente a la sociedad huésped, al mismo tiempo son críticos y contestatarios frente a su país de origen, lo que justifica su activismo político (Calderón y Martínez, 2002: 42).

\section{Metodología y análisis de la encuesta}

La encuesta del Pew Hispanic Center en 2006, ${ }^{5}$ mostró similares porcentajes sobre tenencia de credencial de elector, de MCAS y de interés en votar a la encuesta levantada en el sur de California (véase tabla 1).

Tabla 1. Porcentajes de credenciales de IFE (para votar), matrículas consulares e interés en votar 2006-2011-12

\begin{tabular}{|c|c|c|c|}
\hline Año & \% IFE & \% no Matricula & \% interesados \\
\hline 2006 & 31 & 52 & $78^{*}$ \\
\hline $2011-12$ & 31 & 54 & 85 \\
\hline
\end{tabular}

Los mexicanos estaban conscientes de que viviendo en Estados Unidos podían votar. Fuente: Elaboración propia con datos de Survey of Mexican Living in the U.S. on Absentee Voting in Mexican Elections, y la Encuesta sobre Credenciales de Elector y Matrículas Consulares de Migrantes en el Sur de California 2011-12.

Los resultados de nuestro estudio comparados con los de la encuesta levantada seis años atrás, muestran porcentajes menores a las estimaciones del IFE sobre los mexicanos que pueden votar. Luis Carlos Ugalde, anterior presidente consejero del IFE, contemplaba a 4.2 millones mexicanos con credencial de elector en $2005,{ }^{6}$ cifra que representa alrededor del 40 por ciento, según el número total de connacionales residiendo en Estados Unidos en aquel entonces (Suro y Escobar, 2006). Estos datos son resultado de una encuesta sesgada que contemplaba a los que solicitaban matrículas

5 La encuesta de Pew Hispanic Center se levantó del 16 de enero al 6 de febrero de 2006. Fueron 987 entrevistas hechas por teléfono a nivel nacional, una muestra estadísticamente representativa. El margen de error fue de $+/-4.51$ por ciento. El estado de California estuvo incluido en la muestra por ser una región de alta concentración de mexicanos. Consultar http://www. pewhispanic.org/2006/02/22/pew-hispanic-center-survey-of-mexicans-living-in-the-us-onabsentee-voting-in-mexican-elections/ (Revisado el 1 de marzo de 2012).

6 Estos datos son resultados de una encuesta realizada entre Julio de 2004 y enero de 2005 a cerca de 5000 mexicanos que solicitaban la matrícula consular en 7 consulados mexicanos alrededor de todo Estados Unidos. 
consulares, y tal como se encontró en la encuesta de 2006 y 2012, más de la mitad de los entrevistados no tiene matrícula.

La selección de los sesenta y ocho entrevistados fue aleatoria en tres eventos. El primero en la ciudad de San Marcos el 25 de septiembre de 2011, al norte del condado de San Diego, California, durante la celebración de la décimo sexta edición de la Guelaguetza y el segundo, en la ciudad de San Diego el 25 de febrero, durante la Semana Binacional de Salud. En ambos eventos se colocó una mesa de información de la Asociación de Michoacanos Andarani de San Diego y Amigos (AMASDA). Se encuestó a quienes se acercaron voluntariamente a la mesa y accedieron a ser entrevistados. El tercer evento tuvo lugar en la ciudad de Los Ángeles el 26 de febrero de 2012, durante una reunión de la Federación de Chiapanecos en Los Ángeles. Ahí, el Instituto Binacional de las Fronteras impartió el taller sobre profesionalización de organizaciones de migrantes y a los asistentes se les pidió participar en la encuesta.

La encuesta como metodología de estudios de caso busca generar hipótesis por medio de la comprensión de contextos y procesos, así como la relación entre causas y resultados (Flyvbjerg, 2011: 314). Propone observar casos críticos que tienen importancia estratégica para el problema en general y permiten conseguir información para hacer deducciones de tipo lógicas, sí esto es válido (no es válido) para este caso, entonces aplica para todos (ninguno) los casos (Flyvbjerg, 2011: 307). Si estas condiciones se presentan en dichos inmigrantes mexicanos, es muy probable que se presenten en todos.

Las personas encuestadas en el sur de California representan casos críticos. Para 2010, el 38 por ciento de los inmigrantes mexicanos adultos vivía en California (Gutiérrez, Batalova y Terrazas, 2012). Esta es la región del país con más clubs de oriundos mexicanos, agrupaciones preocupadas por incidir en los asuntos públicos que les conciernen en ambos lados de la frontera y que ayudan a tender puentes de comunicación entre las comunidades migrantes y las autoridades políticas de sus regiones de origen. Por otro lado, hay cinco consulados que atienden a la comunidad mexicana en la región. ${ }^{7}$ Los consulados han sido el vínculo entre la comunidad y el IFE, pues ahí han llegado las hojas de registro a la Lista Nominal de Electores Residentes en el Extranjero (LNERE) y desde luego, por su condición de

7 California es el segundo estado con más consulados (10) después de Texas (11). En el sur de California se encuentran los de Calexico, Fresno, Los Ángeles, Oxnard, San Bernardino, San Diego y Santa Ana. Hay otros tres consulados en el norte de California, en Sacramento, San Francisco y San José. 
frontera que posibilita, a quienes pueden, regresar a solicitar la credencial de elector en México. Debido a su ubicación geográfica, los mexicanos del sur de California tendrían mejores posibilidades de contar con credenciales de elector y/o matrículas consulares.

\section{Resultados de la encuesta}

De los sesenta y ocho encuestados, el 41 por ciento no sabía que podía votar por presidente en 2012, a pesar de que el 72 por ciento dijo contar con internet. Sin embargo, el 85 por ciento mencionó que sí votaría en las elecciones. Así mismo, al 95 por ciento le interesan los asuntos en México. Cuarenta y dos personas mandan dinero a México, dieciocho de ellos por lo menos una vez al mes, de los cuales diez no tiene credencial de elector.

\section{Gráfica 1. Porcentajes con y sin credencial de IFE entre hombres y mujeres de la encuesta}

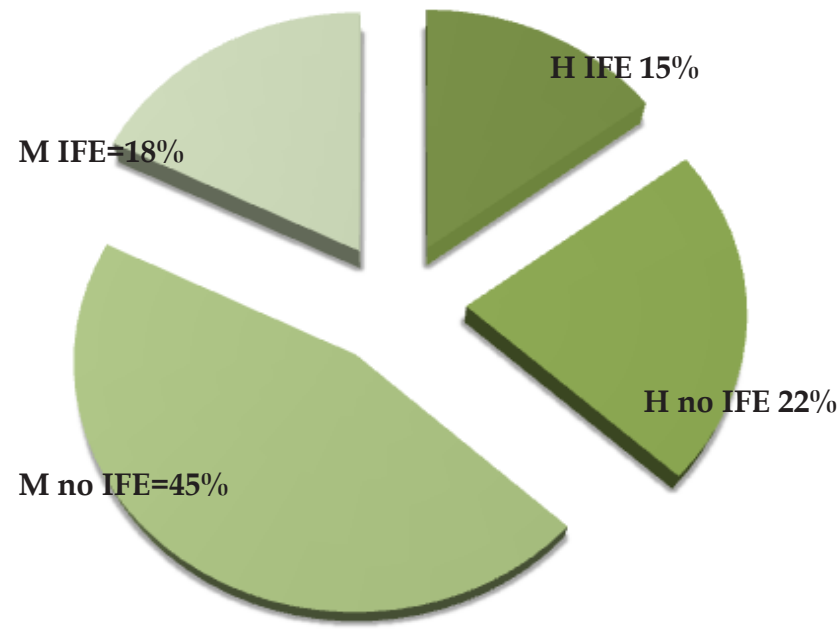

Fuente: Elaboración propia con datos de la Encuesta sobre Credenciales de Elector y Matrículas Consulares de Migrantes en el Sur de California, 2011-2012.

Como muestra la tabla 2, las personas sin credencial de elector tienden a ser más jóvenes y llegaron a Estados Unidos a una edad más temprana, -una tercera parte antes de cumplir los dieciocho años. Al mismo tiempo, los años de residencia no tienen una relación positiva con la tenencia de credenciales de elector; es decir, no por tener más años de residencia, losresidentes mexicanos tienen credencial de elector. Tener Internet tampoco 
se relaciona positivamente con tener credencial, a pesar de que a través de la red también se puede uno inscribir para votar.

Tabla 2. Características de personas con y sin credencial de elector

\begin{tabular}{|c|c|c|c|c|c|}
\hline $\begin{array}{c}\text { credencial } \\
\text { IFE }\end{array}$ & $\begin{array}{c}\text { Media } \\
\text { de } \\
\text { edad }\end{array}$ & $\begin{array}{c}\text { Media } \\
\text { de } \\
\text { residencia }\end{array}$ & $\begin{array}{c}\text { Media de edad } \\
\text { cuando llegaron } \\
\text { al país }\end{array}$ & $\begin{array}{c}\text { Porcentaje con } \\
\text { internet }\end{array}$ & $\begin{array}{c}\text { Porcentaje llegaron antes } \\
\text { de los 18 años }\end{array}$ \\
\hline Con & 45 & 17 & 27 & 63 & 9 \\
\hline $\operatorname{Sin}$ & 38 & 19.7 & 18.3 & 73 & 34 \\
\hline
\end{tabular}

Fuente: Elaboración propia con datos de la Encuesta sobre Credenciales de Elector y Matrículas Consulares de Migrantes en el Sur de California, 2011-2012.

Para el caso de las matrículas consulares, tenemos que de 37 personas, 54 por ciento, no cuenta con una, mientras que 25 personas, 37 por ciento, sí poseen dicho documento. ${ }^{8}$ Por género, encontramos que 60 por ciento de los hombres no tiene matrícula al igual que el 60 por ciento de las mujeres. Es decir, la mayoría de hombres y mujeres encuestados no cuentan con el documento que el gobierno mexicano ha diseñado para identificar a los connacionales en Estados Unidos.

\section{Gráfica 2. Porcentajes con y sin matrículas consulares entre hombres y mujeres de la encuesta}

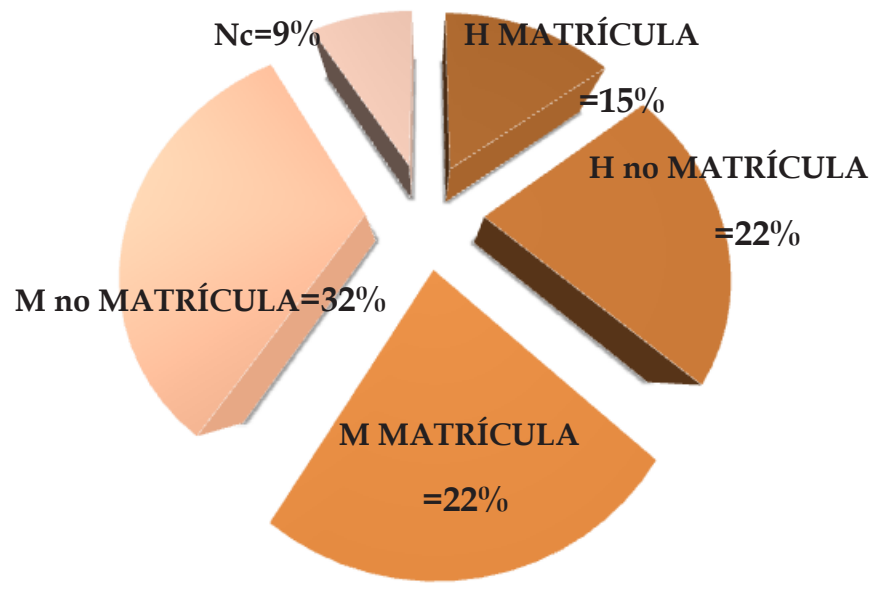

Nota: De todas las que no contestaron sobre la matricula consular, todas fueron mujeres.

Fuente: Elaboración propia con datos de la Encuesta sobre Credenciales de Elector y Matrículas Consulares de Migrantes Mexicanos en el Sur de California, 2011-2012.

8 El porcentaje faltante no contestó a la pregunta de si tenía matrícula consular. 
La mayoría de quienes no tienen credencial de elector, tampoco tiene matrículas consulares. De los cuarenta y dos encuestados sin credencial para votar, veintisiete no tienen matrículas. De veinte personas con credencial para votar, sólo diez tienen matrículas (véase gráfica 3). Lo que se puede concluir de la relación entre credenciales y matrículas, es que los encuestados no suplieron la falta de credenciales con éstas últimas. De los sesenta y ocho encuestados sólo diez tienen ambas. Por otro lado, de los sesenta y ocho encuestados, veintisiete no tienen ninguna de las dos.

\section{Gráfica 3. Relación entre credenciales de elector y matrículas}

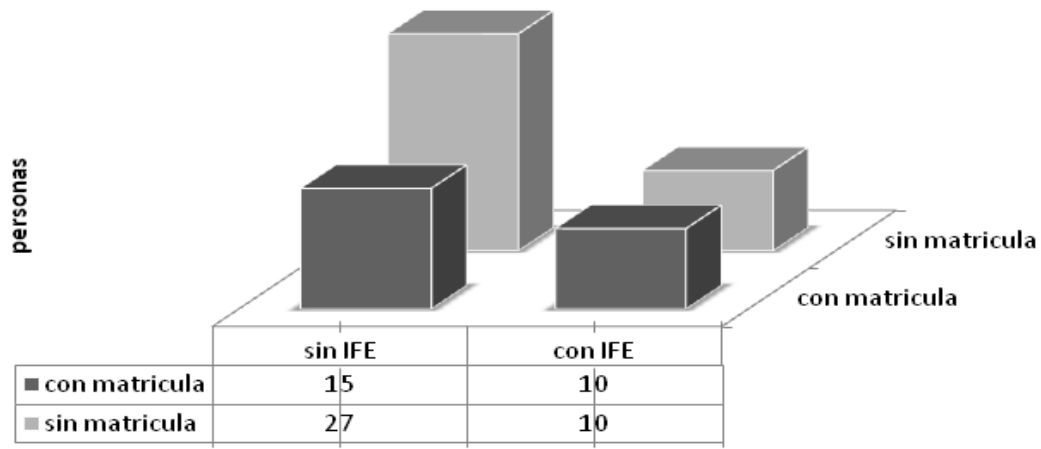

Nota: Los 6 faltantes para completar los 68 encuestados, no contestaron si tenían matrícula, por eso fueron excluidos de esta relación.

Fuente: Elaboración propia con datos de la Encuesta sobre Credenciales de Elector y Matrículas Consulares de Migrantes Mexicanos en el Sur de California, 2011-2012.

En lo que se refiere a educación, no hay una relación positiva entre mayor nivel de educación y más credenciales de elector (véase tabla 3). 
Tabla 3. Relación entre niveles de educación y falta de credenciales IFE

\begin{tabular}{|c|c|c|c|}
\hline Instrucción & \% total sin credencial & \% mujeres sin credencial & \% hombres sin credencial \\
\hline Primaria & 57 & 50 & 66 \\
\hline Secundaria & 75 & 85 & 66 \\
\hline Preparatoria & 65 & 66 & 66 \\
\hline Universidad & 57 & 33 & 75 \\
\hline
\end{tabular}

Fuente: Encuesta sobre Credenciales de Elector y Matrículas Consulares de Migrantes 2011-2012

Los porcentajes de matrículas consulares son similares a los encontrados en la encuesta del Pew Hispanic Center de 2006, es válido entonces preguntarse, ¿la matricula consular podría sustituir a la credencial de elector como instrumento para poder votar en Estados Unidos, aún considerando que no todos los estados de la Unión Americana la aceptan como identificación oficial y que además, se ha convertido en una credencial que principalmente identifica a los indocumentados?.

\section{¿Por qué ampliar las posibilidades de votar?}

En 1998, según el Informe Final de la Comisión de Especialistas que Estudia las Modalidades del Voto de los Mexicanos en el Extranjero, se concluyó que para el año 2000, 1.5 millones de mexicanos podían votar desde Estados Unidos. La investigación fue llevada a cabo por el Consejo General del IFE después de realizar encuestas de flujos migratorios, ${ }^{9}$ algunas de las cuales se realizaron en la franja fronteriza y en aeropuertos dejando fuera a millones de mexicanos indocumentados. En 1998, la comisión antes citada estimaba que en el año 2000 habría 2.7 millones de connacionales "en situación jurídica migratoria irregular", de los cuales el 25 por ciento de ellos serían votantes potenciales. ${ }^{10}$ La realidad rebasó las proyecciones de la comisión. Para el año 2000, se estimaba que había 4 millones 680 mexicanos indocumentados (Hoefer, Rytina y Baker, 2010).

La Comisión concluyó que técnicamente, la participación de los mexicanos en el exterior era viable para las elecciones presidenciales de 2000 y se contemplaba que votarían: a) los residentes en México: a.1) los mexicanos

9 No se revela específicamente a qué encuestas se refieren.

10 Informe final de la comisión de especialistas que estudia las modalidades del voto de los mexicanos en el extranjero. Resumen ejecutivo. Consejo General del Instituto Federal Electoral. http://www.huellasmexicanas.org/alejandra/voto/InformeVoto-Mexicanos-Extranjero.pdf (Revisado el 1 de febrero de 2012), 
residentes en México durante la jornada electoral; a.2) los mexicanos residentes en México ausentes del país en la misma fecha de las elecciones. A su vez, éstos se catalogaban en a.2.1) los que están en el exterior por turismo; a.2.2) los migrantes temporales; a.2.3) los trasmigrantes o computers; b) los mexicanos radicados en el extranjero. A su vez, éstos se dividían en b.1) los residentes en Estados Unidos, mismos que se subdividían en: b.1.1) los funcionarios del Servicio Exterior mexicano; b.1.2) los inmigrantes nacidos en México; b.1.3) las personas que nacieron en Estados Unidos y son hijos de padre o madre mexicana o ambos padres y por último; b.2) los mexicanos radicados en otros países. ${ }^{11}$

En esta clasificación de votantes, los mexicanos indocumentados se ubican en la subcategoría b.1.2) inmigrantes nacidos en México, y no se les otorga una consideración especial. Aún cuando sus realidades políticas son diferentes, se les considera igual que a los residentes legales, obviando una realidad importante de la migración para aquellos años, la población indocumentada mexicana iba en aumento. Así mismo, llama la atención la falta de visión al no contemplar las acciones antiinmigrantes que ya se venían gestando desde algún tiempo en contra de los indocumentados. Desde la amnistía de 1986 las políticas antiinmigrantes dirigidas a indocumentados ya se estaban desarrollando.

También es de notar que la Comisión que levantó las encuestas para determinar el número de credenciales de elector, no buscara el apoyo de los clubs de oriundos que tenían importante presencia en estados como California. Tan sólo en la ciudad de Los Ángeles, existían 170 clubs de oriundos de dieciocho estados mexicanos registrados ante el consulado mexicano (Zabin y Escala, 1998) para cuando se hizo el estudio. ${ }^{12}$

Las inexactitudes de las estimaciones provocaron la gran diferencia entre lo que se proyectaba y lo que sucedió. En 1998, el IFE pensaba que, en el año 2000, 1.5 millones de mexicanos podían votar. En las elecciones de 2006, cuando ya fue posible votar, sólo 28,335 votos de mexicanos llegaron desde Estados Unidos (Calderón, 2007: 12). Seis años después, en los comicios del 2012, el voto desde Estados Unidos aumentó marginalmente, sobre todo si se considera el tamaño de la comunidad mexicana (véase tabla 4$).{ }^{13}$

11 Informe Final de la comisión de especialistas que estudia las modalidades del voto de los mexicanos en el extranjero. Resumen ejecutivo. Consejo General del Instituto Federal Electoral. Pp. 8 y 9. http://www.huellasmexicanas.org/alejandra/voto/InformeVoto-Mexicanos-Extranjero. pdf (Revisado el 1 de febrero de 2012).

13 Los votos emitidos desde el resto del mundo tuvieron un incremento de alrededor del 20 por ciento y los de Estados Unidos de casi 3.5 por ciento. En 2012, el mayor número de votos des- 
La principal causa de esta situación es la falta de credenciales de elector; el requerimiento de la credencial con fotografía que sólo es posible obtener acudiendo en persona a los lugares de emisión dentro del país, fue considerado como un elemento que provocó la poca participación en México (Navarro, Morales y Gratschew, 2007: 32).

\section{Tabla 4. Inscripción a la lista nominal, votos enviados desde el exterior y desde Estados Unidos 2006 y 2012}

\begin{tabular}{|l|l|l|l|}
\hline $\begin{array}{l}\text { Elección } \\
\text { presidencial }\end{array}$ & $\begin{array}{l}\text { Inscripción a la } \\
\text { LNERE }\end{array}$ & Votos enviados & Votos desde Estados Unidos \\
\hline 2006 & 54780 & 32632 & 28335 \\
\hline 2012 & 56687 & 40737 & 29348 \\
\hline
\end{tabular}

Fuente. Elaboración propia con datos de IFE. ${ }^{14}$

El voto desde el extranjero es un derecho de muchas democracias en el mundo. ${ }^{15}$ Éste ha sido ganado por los que desde Estados Unidos siguen interesados en los asuntos políticos de México y demuestra que la democracia, más que un punto de llegada, es un camino sobre andamios inestables que se deben ajustar y asegurar para que duren. El voto nunca es concedido ni asegurado de una vez por todas, sino que a menudo es revocado, su ejercicio sometido a abusos y atropello y su traducción en las casillas manipulada con fraudes y chanchullos (Pasquino, 2011: 80).

\section{Discusión}

¿Por qué ampliar las posibilidades de votar a los 11.9 millones ${ }^{16}$ de mexicanos residentes en la Unión Americana? La respuesta está en las características inherentes a la migración: es longeva (importante desde hace más de un siglo) y grande por los individuos involucrados. ${ }^{17}$ Entre 2000 y 2010, un

de Estados Unidos, llegó de California (Gutiérrez, Btalova y Terrazas, 2010).

http://www.ife.org.mx/documentos/proceso_2005-2006/cuadernos/pdf/C4/c4_4-1.pdf. http:// www.votoextranjero.mx/documents/10157/4fe7cd4d-8e67-487a-8b60-26ce0894e874. (Revisado el 15 de enero de 2012).

15 Para 2007, 115 países en el mundo permitían votar a sus ciudadanos desde el extranjero. http:// www.idea.int/publications/voting_from_abroad/upload/Voting_from_abroad.pdf (Revisado el 15 de enero de 2012).

16 Número estimado por CONAPO 2010. http://www.conapo.gob.mx/publicaciones/intensidad_migratoria/texto/Migracion_Mex_EU.pdf (Revisado el 15 de marzo de 2012).

17 México es el país de mayor inmigración a la Unión Americana. Consejo Nacional de Población, CONAPO. http://www.conapo.gob.mx/publicaciones/intensidad_migratoria/texto/Migra- 
estimado de 1.62 millones de hombres y 1.14 millones de mujeres, buscaron destinos en el extranjero, casi todos en Estados Unidos (Campos-Vázquez y Sobarzo, 2012). Además, se tiene que considerar que la gran mayoría de los mexicanos decidieron partir al norte evaluando condiciones de vida, trabajo, ingreso, progreso personal y familiar. Estos asuntos tienen estrecha relación con políticas económicas y éstas a su vez con la distribución planeada o no de los recursos; en cómo las élites deciden las políticas públicas.

Bajo este escenario de migración sur-norte, consideramos que existen condiciones propicias para ampliar las posibilidades reales de votar desde el extranjero. Tres factores que deben tomarse en cuenta: a) la transición democrática, b) el crecimiento de los clubs de oriundos y su interés por los asuntos públicos en sus lugares de origen y c) el interés del gobierno federal y gobiernos subnacionales por los migrantes.

Primero. En México, la discusión sobre los asuntos públicos se enriqueció con la pluralidad que trajo la transición democrática, con más énfasis desde los años noventa del siglo pasado. Concretamente, significó que más individuos pudieran tomar decisiones políticas. Se estima que un 10 por ciento de los nacidos en México reside en el vecino país del norte y una parte de ellos reclamó su espacio en esa nueva pluralidad. Este interés político se vio alentado por el coqueteo de los políticos profesionales mexicanos. Desde que el ingeniero Cuauhtémoc Cárdenas visitó a la comunidad mexicana en Los Ángeles en 1988 durante su campaña presidencial, los partidos políticos mexicanos han volteado al norte en busca de simpatiza.

Las elecciones democráticas federales organizadas por el IFE y en el caso de la elección presidencial, calificada por el Tribunal Electoral del Poder Judicial de la Federación (TEPJF), son una novedad para quienes dejaron un país de injusticia electoral, donde un partido hegemónico aseguraba para sí triunfos electorales, recursos y bienes públicos. Para los migrantes mexicanos que dejaron el país desde el siglo pasado, las elecciones pueden ser vistas como la oportunidad para participar en los asuntos públicos.

El voto es la forma primordial de participación política, el hecho más relevante para elegir a quién deberá decidir (Bobbio, 2009: 402), pues permite someter a los políticos profesionales al escrutinio de los gobernados (Del Águila, 2009: 141). Las elecciones que introducen a los gobernados a la discusión de hacia dónde se van los recursos públicos de manera pacífica, deben ser un proceso incluyente en un sistema en construcción democrática, ya que aquello que distingue a la democracia de otras formas de convivencia política es la igualdad (Bovero, 2002: 18). 
Segundo. La organización político-social de los migrantes en las ciudades de destino a través de los clubs de oriundos va en aumento, se trata de organizaciones cada vez más interesadas por asuntos públicos en los lugares de origen. Esto se puede medir a través del creciente número de proyectos de inversión y los montos que estos clubs hacen en México por medio del programa $3 \times 1$, éste es manejado a través de la Secretaría de Desarrollo Social (SEDESOL) y busca colectivizar las remesas y que los migrantes organizados propongan pautas de desarrollo local, principalmente en inversión en infraestructura, obra pública, social y proyectos productivos. Los clubs de migrantes residentes en el extranjero proponen inversiones y el gobierno municipal (de dónde provienen los integrantes del club), el gobierno estatal y el gobierno federal aportan una cantidad similar para financiar los proyectos. Este programa se convierte en uno de los principales instrumentos de la interacción del Estado mexicano con la comunidad migrante en Estados Unidos (García, 2005:12). La inversión de los clubs a través del programa $3 \times 1$ pasó de $\$ 97,416,029$ pesos en 2003 a $\$ 407,036,984$ en 2012. Es decir, se cuadruplicó en nueve años (SEDESOL. Programa $3 \times 1$ para migrantes).

Independientemente de los problemas de organización interna, representatividad, coordinación, movilización o participación política (Escala, Rivera-Salgado y Rodríguez, 2011), los clubs de oriundos representan un fuerte vínculo entre la comunidad migrante y sus lugares de origen y suelen establecer relaciones políticas con las autoridades locales mexicanas. Son una expresión de la organización política para discutir asuntos públicos. Algunos han demostrado mayor actividad social y política frente a los embates que llegan desde los políticos y nativos antiinmigrantes. En 1994 mostraron haber jugado un rol activo durante la batalla política contra la proposición 187, que pretendía negar servicios sociales, médicos y educación pública a los indocumentados en California.

A través de estas organizaciones, los inmigrantes han ganado poder político local en sus lugares de residencia al mismo tiempo que en las regiones de las cuales emigraron, contribuyendo a la construcción de instituciones democráticas (Zabin y Escala, 1998). Estos clubs de oriundos siguen teniendo una mayor presencia en el sur de California, ahí se concentran cientos de clubs formados por migrantes. En 1998, había 170 clubs mexicanos en Los Ángeles y su número creció a 420 en 2005. El Frente Indígena de Organizaciones Binacionales (FIOB), es un claro ejemplo de cómo las identidades étnicas funcionan para la organización política. Fue establecido en 1994 como Frente Indígena Oaxaqueño Binacional, por migrantes indígenas mexicanos en Estados Unidos y Baja California. Promueve de 
manera binacional, la autodeterminación de los pueblos indígenas, la defensa de los derechos humanos, la justicia y la igualdad de género (Perry et al., 2009; Martínez, 2012: 150); entre muchas otras acciones, el FIOB promovió la participación en las elecciones presidenciales de 2012. ${ }^{18}$

El aumento del interés de los clubs en los asuntos de sus lugares de origen, ha ido acompañado con el creciente interés del gobierno federal y los gobiernos subnacionales por las comunidades migrantes. Estos últimos han sido importantes promotores de la organización comunitaria.

Tercero. El gobierno mexicano y los gobiernos subnacionales ven al inmigrante como sujeto político, como partícipe de la construcción de la sociedad aunque no se encuentre físicamente en ella. Es el estado que impulsa la migración transnacional (Roberts, Reanne y Lozano-Ascencio, 2003: 68). El gobierno federal y los gobiernos subnacionales han desarrollado políticas públicas donde los inmigrantes son partícipes del crecimiento económico y desarrollo local por el substancial incremento de las remesas en los últimos años (Alba, 2009). ${ }^{19}$ En algunos estados, el monto de las remesas supera la inversión extranjera directa (Correa, 2008, Campos-Vázquez y Sobarzo, 2012). ${ }^{20}$

Desde principios de los años noventa, el Estado mexicano ha implementado un acercamiento activo hacia los mexicanos en el extranjero. Desde la administración Salinista (1988-1994) se incrementaron las oficinas consulares y se asignaron más recursos para atender a los mexicanos que residían en el exterior a través de la creación de nuevos programas (Zabin y Escala, 1998) tales como el Programa de Apoyo a las Comunidades Mexicanas en el Extranjero, Solidaridad Internacional y el programa Paisano (Calderón y Martínez, 2002: 53).

Para 2010, veinticuatro de treinta y dos entidades en México tenían instituciones para atender a sus comunidades en el exterior (Yrizar y Alarcón, 2010: 166). El Instituto de los Mexicanos en el Exterior, IME, creado en 2003, estrechó relaciones del Estado mexicano con sus migrantes. Algunas en-

18 Frente Indígena de Organizaciones Binacionales, http://fiob.org (Revisado el 20 de marzo de 2012).

19 México es el mayor receptor de remesas en América Latina y el tercero en el mundo, con incrementos vertiginosos entre 2000 y 2007, de \$6,500 a casi \$25,000 millones de dólares. Sin embargo, esta tendencia puede haber entrado en una fase de estancamiento. Para 2010, el total de remesas alcanzaron los \$22 mil millones de dólares, el 2.5 por ciento del PIB.

20 En Guerrero, la Inversión Extranjera Directa (IED) representa menos del 1 por ciento del Producto Interno Bruto del estado, mientras que las remesas casi el 15 por ciento, en Oaxaca la IED menos de 1 por ciento y las remesas casi el 10 por ciento del PIB. Sólo Baja California, Baja California Sur, Colima, Distrito Federal, Nuevo León y Querétaro, superan la IED a las remesas como porcentaje del PIB. 
tidades han ido a la delantera en la misma dirección: Michoacán permite el voto desde Estados Unidos para elegir gobernador y el Distrito Federal para elegir jefe de gobierno. El estado de Zacatecas contempla la elección de dos migrantes de entre doce diputados plurinominales elegidos por el principio de representación proporcional. ${ }^{21}$

Las entidades subnacionales han tomado en cuenta el número de sus ciudadanos en Estados Unidos para replantear la importancia de sus migrantes. Para el conteo poblacional de 2005, 35 por ciento de los zacatecanos vivían en Estados Unidos, al igual que el 25 por ciento de los michoacanos y el 24 por ciento de los duranguenses (Yrizar y Alarcón, 2010: 178).

La democratización del sistema, el incremento de los clubs de oriundos y su creciente interés por asuntos públicos a través del aumento de sus inversiones en México, así como el interés del gobierno federal y los gobiernos subnacionales por los migrantes, pueden provocar, tomando las medidas adecuadas, un substancial incremento en la participación electoral de la comunidad inmigrante en Estados Unidos. Sin embargo, la falta de credenciales de elector limita esta posibilidad.

Ejercer el voto en el país que dejaron, es la única forma de participación política para la mayoría de los migrantes, a muchos de ellos les importa ejercerlo porque es el país al cual regresarán, o a donde probablemente los regresen, al que mandan dinero, en el que tienen parientes y amigos, en el cual ayudan a construir infraestructura o crear empleos y al que continuamente festejan.

El poco crecimiento de los votos desde Estados Unidos podría entenderse por el escaso interés que tienen los inmigrantes por los asuntos políticos en México. Sin embargo, se ha encontrado en diversos estudios un importante interés en los asuntos de México entre la comunidad inmigrante, tal como lo muestran los resultados de la encuesta del Pew Hispanic Center y la encuesta en el sur de California, o la que levantó el Proyecto de Investigación de Campo de la Migración Mexicana y Programa de Entrenamiento, Mexican Migration Field Research and Training Program (MMFRP) de la Universidad de California, San Diego, antes de las elecciones de 2006 en Tunkás, Yucatán, demostrando que la migración no necesariamente aleja a los individuos del interés por los asuntos públicos de los lugares de origen. Por ejemplo, el 76 por ciento de los migrantes de Tunkás, declaró que votaría en esas elecciones. Sin embargo, como índica el propio estudio, redactado después de las elecciones de julio, el número

21 Reforma al Artículo 51 de la Constitución política del estado libre y soberano de Zacatecas. 
de votos emitidos desde Estados Unidos sugiere que sólo un puñado de tunkaseños votaron (Ruiz et al., 2007: 241).

También Alarcón et al. (2012), encuentran que si bien la comunidad mexicana en Los Ángeles no participó en las elecciones de 2006, los mexicanos manifestaron estar informados de la política mexicana y la percibieron como corrupta e incapaz de gobernar, mostrando particular interés en el tema de la violencia. De esto se concluye que hay una creciente inclinación de los inmigrantes mexicanos por participar en la esfera política y particularmente en el acontecer político en los lugares destino a raíz de los embates antiinmigrantes (Alarcón et al., 2012: 303).

En el proceso de transición democrática se sigue marginando a amplios sectores de la sociedad, como a muchos de los mexicanos migrantes que no tienen credencial de elector. De 6 millones 640 mil mexicanos indocumentados residentes en Estados Unidos en la actualidad y 2 millones 570 mil en California, el 87 por ciento, según estimaciones, son mayores de dieciocho años (Hoefer, Rytina y Baker, 2010). Para estos migrantes, los costos y riesgos del cruce clandestino limitaron la circularidad de épocas pasadas y colateralmente el derecho a sufragar.

\section{Conclusiones}

Existe un escenario propicio para fomentar la participación electoral de los migrantes que no se ha aprovechado. La escena de transición democrática donde los partidos de oposición ganan continuamente elecciones locales y la alternancia es una realidad, donde las elecciones suelen ser más legales, transparentes y justas que en épocas pre democráticas, donde los clubs de oriundos, expresión de organización política de los migrantes, va en aumento al igual que sus inversiones públicas en sus lugares de origen. A este escenario lo completa el interés del gobierno federal y gobiernos subnacionales por los migrantes a través de diversas propuestas como el Programa $3 \times 1$ para migrantes.

La modalidad del voto postal puede funcionar mejor si se revisan los procedimientos en los que se sustenta. No sólo es importante considerar que una tercera parte de los migrantes tiene credencial para votar, sino también repensar en los procedimientos técnicos y administrativos que limitaron el voto en 2006. Para esto, hay que tomar en cuenta el alcance que los consulados tienen para distribuir las solicitudes de inscripción, el costo de la modalidad del voto por correo postal certificado, el llenado, complicado 
para muchos, de las solicitudes de inscripción a la lista nominal, la escasa organización en la distribución de solicitudes, etcétera.

También se debe reconsiderar el papel de las organizaciones comunitarias, entre ellas los clubs de oriundos, para distribuir las solicitudes de registro a la lista nominal, pues los consulados se vieron rebasados en la entrega de solicitudes. En 2006, en el consulado de México en Chicago, se restringió la entrega de solicitudes de inscripción a una por cada persona que la solicitara personalmente en el consulado, esto implicaba el traslado del solicitante. Este consulado da servicio a todo Illinois, al sur de Wisconsin y al norte de Indiana. Aunado al costo de enviar el correo postal, que en aquel entonces era de $\$ 8$ dólares, el votante potencial tenía que considerar los costos de traslado desde su lugar de residencia al consulado de Chicago y en algunos casos los costos se elevaban considerablemente. El costo de la modalidad postal redujo mucho el interés.

Lo que vemos, considerando la encuesta hecha por el Pew Hispanic Center, es que los costos de traslados pudieron haber afectado el interés por votar en diversas regiones del país. Así también, nuestra encuesta ayuda a ver que en el sur de California, seis años después, con cinco consulados mexicanos, los costos para los votantes potenciales pueden seguir siendo un factor que limita la intención del voto.

La migración de mexicanos a Estados Unidos, en constante transformación, es un mosaico de realidades que requieren análisis geográficamente diferenciados. No es la misma migración a Illinois que a California, o los nuevos destinos como Carolina del Norte. Debido a esta diversidad de realidades se puede pensar en una diversidad de modalidades de voto.

Otro asunto que debe considerarse en la discusión es el referente a los costos. En 2006, no todos los recursos destinados para el voto se ejercieron; para 2012, el IFE reportó en su informe final que logró incrementar el número de votos disminuyendo los costos, ${ }^{22}$ pues se tomaron medidas pertinentes desde la experiencia anterior. Esto hace pensar que el incremento de los votos no necesariamente aumentaría el gasto de forma sustancial. Tomando en cuenta las limitaciones presupuestales necesarias, este voto se puede seguir fomentando. El seguir pensando en modalidades del voto

22 El Consejo General del IFE aprobó un presupuesto de 264.1 millones de pesos. De estos recursos no fueron ejercidos, al término del ejercicio 2005, \$144.8 millones de pesos del presupuesto autorizado para el Voto de los Mexicanos Residentes en el Extranjero, por lo que se procedió al reintegro a la Tesorería de la Federación (TESOFE). Revisado el 1 de septiembre de 2012. http://mxvote06.ife.org.mx/libro_blanco/pdf/tomoVI/tomoVI.pdf 
es precisamente lo que lleva a considerar posibilidades que disminuyan su costo. ${ }^{23}$

La credencial para votar con fotografía con elementos de seguridad fue una innovación, un resultado de nuestra historia de desconfianza política que muy difícilmente cambiará a corto plazo. Se inicia un período de seis años, ¿por qué no seguir innovando hasta el registro para votar desde el extranjero en 2018 ?

\section{Bibliografía}

Alarcón Acosta, Rafael, Luis Escala Rabadán y Olga Odgers Ortiz, 2012, Mudando el hogar al norte. Trayectorias de integración de los inmigrantes mexicanos en Los Ángeles, México, El Colegio de la Frontera Norte.

Alba, Francisco, 2009, “Migración Internacional y Políticas Públicas”, en El Estado de la Migración. Las Políticas Públicas ante los retos de la migración mexicana a Estados Unidos, México, Consejo Nacional de Población, pp. 23-89.

Bobbio, Norberto, 2009, Teoría general de la política, España, Editorial Trotta.

Bovero, Michel Angelo, 2002, Una Gramática de la democracia. Contra el gobierno de los peores, España, Editorial Trotta.

Calderón Chelius, Leticia, 2007, “En busca del voto perdido: Análisis de los resultados del voto en el exterior en la elección presidencial mexicana de 2006", en Cecilia Imaz Bayona (coord.), ¿Invisibles? Migrantes internacionales en la escena política, México, Facultad de Ciencias Políticas UNAM, pp. 199-214.

Calderón Chelius, Leticia y Jesús Martínez Saldaña, 2002, La dimensión Política de la migración mexicana, México, Instituto de Investigaciones Dr. José María Luis Mora.

Campos-Vázquez Raymundo y Horacio Sobarzo, 2011, The Development and Fiscal Effects of Emigration on Mexico, Washington, DC, Woodrow Wilson International Center for Scholars, Migration Policy Institute. http://www.migrationpolicy. org/pubs/RMSG-fiscaleffects-emigration.pdf (Revisado el 10 de octubre de 2012).

23 El costo presupuestal de cada voto desde el extranjero fue de \$12 mil 582, aproximadamente mil dólares. 
Colomer, Josep M., 2004, Cómo votamos. Los sistemas electorales del mundo: pasado, presente y futuro, España, Editorial Gedisa.

Correa Alcantar, Manlio César, 2006, “The economic potential of collective remittances: an examination of the current and potential impact of Mexican migrant hometown associations", en José Rodolpho Jacobo y Alex Gomez (eds.), Frontera Del Norte: Readings in Chicano and Border History, United States, Southern Border Press, pp. 239-270.

Del Águila, Rafael, 2009, “La Democracia, Manual de Ciencia Política”, en Rafael del Águila (ed.), Manual de Ciencia Política, España, Editorial Trotta.

Emmerich, Isaac, Gustavo Ernesto y Carlos Moreira, 2013, “Sufragio transnacional desde el exterior y los extranjeros en Argentina", De Política. Revista de la Asociación Mexicana de Ciencias Políticas, año 1, núm. 1, pp. 29-60.

Escala Rabadán, Luis, Gaspar Rivera Salgado y Rigoberto Rodríguez, 2011, “Is More Necessarily Better? Leadership and Organizational Development of Migrant Hometown Associations in Los Angeles, California", Migraciones Internacionales, vol. 6, núm. 2, pp. 41-73.

Flyvbjerg, Bent, 2011, “Case Study”, en Norman K. Denzin e Yvonna S. Lincoln, The Sage Handbook of Qualitative Research, 4th Edition, Thousand Oaks, California, Sage, pp. 301-316.

García Zamora, Rodolfo, 2005, “Las Remesas colectivas y el programa 3 x 1 como proceso de aprendizaje social transnacional", Documento presentado en el seminario La Participación cívica y social de los migrantes mexicanos en Estados Unidos, Washington D.C., 4 y 5 de noviembre.

Grupo Reforma. Informe de la encuesta sobre preferencias electorales en el DF. http://www.ife.org.mx/documentos/proceso 2011-2012/EncuestasConteosRapidos/docs-encuestas/Reforma/junio9-10/Informe.pdf. (Revisado el 1 de febrero de 2013).

Gutierrez, David, Jeanne Btalova y Aarón Terrazas, 2010, “Mexican Presidential Election and Mexican Immigrants of Voting Age in the United States", en Migration Policy Institute. http://www.migrationpolicy.org/article/2012-mexican-presidential-election-and-mexican-immigrants-voting-age-united-states / Revisado el 15 de junio de 2012

Hoefer, Michael, Nancy Rytina y Bryan C. Baker, 2010, “Estimates of the Unauthorized Immigrant Population Residing in the United States", en Homeland Se- 
curity, Office of Immigration Statistics. https://www.dhs.gov/xlibrary/assets/ statistics/publications/ois_ill_pe_2011.pdf. (Revisado el 1 de Julio de 2011).

Huntington, Samuel P., 2004, ¿Quiénes somos? Los desafíos de la identidad nacional estadounidense, Madrid, Editorial Paidós.

Johnson, Kevin R., 1997, "Melting Pot or Ring of Fire: Assimilation and the MexicanAmerican Experience”, California Law Review, vol. 85, núm. 5, pp. 1259-1313.

Levitt, Peggy y Mary C. Waters, 2002, The Transnational lives of the second generation, Rusell Sage Foundation.

Martínez Esparza, Prisca Adriana, 2012, “Prácticas políticas en una organización binacional indígena: el caso del FIOB en Juxtlahuaca", en María Dolores París Pombo (coord.), Migrantes, desplazados, braceros y deportados. Experiencias migratorias y prácticas políticas, México, COLEF, UACJ, UAM.

Navarro Fierro, Carlos, Isabel Morales y María Gratschew, 2007, “External voting: a comparative overview", Voting from Abroad. The International IDEA Handbook, International Institute for Democracy and Electoral Assistance, Federal Electoral Institute of Mexico. http://www.idea.int/publications/voting_from_abroad/ loader.cfm?csmodule=security $/$ getfile\&pageid $=23636$ (Revisado el 1 de junio de 2012).

Pasquino, Gianfranco, 2011, Nuevo curso de ciencia política, México, Fondo de Cultura Económica.

Perry, E., N. Doshi, J. Hicken y J.R. Méndez García, 2009, “Between Here and There: Ethnicity, Civil Participation, and Migration in San Miguel Tlacotepec", en Wayne A. Cornelius, David Fitzgerald, Jorge Hernández-Díaz y Scott Borger (eds.), Migration from the Mexican Mixteca. A transnational Community in Oaxaca and California, Center for Comparative Immigrations Studies, University of California, San Diego.

Portes, Alejandro, Luis Guarnizo y Patricia Landolt, 2003, “El estudio del trasnacionalismo: peligros latentes y promesas de un campo de investigación emergente", en Alejandro Portes, Luis Guarnizo y Patricia Landolt (coords.), La Globalización desde abajo" Transnacionalismo inmigrante y desarrollo. La experiencia de Estados Unidos y América Latina, México, FLACSO.

Portes, Alejandro y Rubén G. Rumbaut, 2010, América Inmigrante, Barcelona, Anthropos. 
Roberts, Bryan, Frank Reanne y Fernando Lozano-Ascencio, 2003, “Las comunidades migrantes transnacionales y la migración mexicana a Estados Unidos" en Alejandro Portes, Luis Guarnizo y Patricia Landolt (coords.), La Globalización desde abajo Transnacionalismo inmigrante y desarrollo. La experiencia de Estados Unidos y América Latina, México, FLACSO.

Ruiz Alonso, Ileana B., Milton Jovanni Sarria and Arturo Severo Vázquez, 2007, "Migration and polítical participation" en Wayne A. Cornelius, David Fitzgerald y Pedro Lewin F. (eds.), Mayan Journeys. The New Migration from Yucatán to the United States, Center for Comparative Immigrations Studies, San Diego, University of California.

Sedesol. Programa $3 \times 1$ para migrantes. http://www.sedesol.gob.mx/es/SEDESOL/Programa_3x1_para_Migrantes. (Revisado el1 de mayo de 2012).

Suro, Roberto y Gabriel Escobar, 2006, "Survey of Mexicans Living in the U.S. on Absentee Voting in Mexican Elections", Washington, D.C., Pew Hispanic Centre.

The Pew Research Center fort the People and the Press, 2012, Assessing the Representativeness of Public Opinion Surveys. http:/ / www.people-press.org/files / legacy-pdf/Assessing\%20the \%20Representativeness \%20of\%20Public\%20Opinion\%20Surveys.pdf (Revisado el 1 de febrero de 2013).

Vertovec, Steven, 2003, “Concebir e investigar el transnacionalismo”, en Alejandro Portes, Luis Guarnizo y Patricia Landolt (coords.), La Globalización desde abajo Transnacionalismo inmigrante y desarrollo. La experiencia de Estados Unidos y América Latina, México, FLACSO.

Yrizar Barbosa, Guillermo y Rafael Alarcón, 2010, “Emigration Policy and State Governments in Mexico", Migraciones Internacionales, vol. 5, núm. 4, pp. 165-198.

Zabin, Carol y Escala Rabadán, Luis, 1998, “Mexican hometown associations and Mexican immigrant political empowerment in Los Angeles", en The Aspen Institute, Working Papers Series.

Recibido el 8 de mayo de 2012 Aceptado el 29 de enero de 2014 